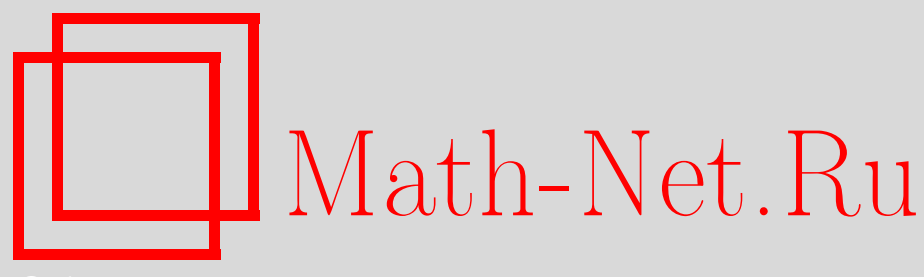

Э. А. Аринштейн, Термодинамическая устойчивость, особые точки и фазовые переходы в теории частичных функций распределения, ТМФ, 2008, том 155, номер 3, 512-523

DOI: https://doi.org/10.4213/tmf6226

Использование Общероссийского математического портала Math-Net.Ru подразумевает, что вы прочитали и согласны с пользовательским соглашением http://www . mathnet.ru/rus/agreement

Параметры загрузки:

IP : 54.166 .219 .16

26 апреля 2023 г., 07:09:12

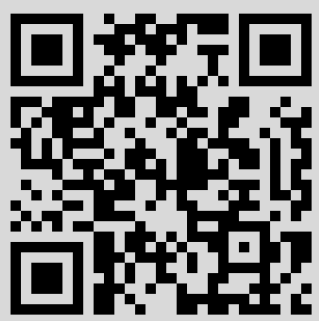




\title{
ФИЗИКА
}

Том 155, № 3

июнь, 2008

2008 г.

Э. А. Аринштейн*

\section{ТЕРМОДИНАМИЧЕСКАЯ УСТОЙЧИВОСТЬ, ОСОБЫЕ ТОЧКИ И ФАЗОВЫЕ ПЕРЕХОДЫ В ТЕОРИИ ЧАСТИЧНЫХ ФУНКЦИЙ РАСПРЕДЕЛЕНИЯ}

\begin{abstract}
Рассмотрена устойчивость минимума термодинамического потенциала как функционала от частичных плотностей или корреляций. Показано, что потеря устойчивости связана с особыми точками термодинамических функций. Линии или точки фазовых переходов первого рода определяются путем сравнения термодинамических потенциалов разных фаз, и условие потери устойчивости по отношению к флуктуациям плотности может служить критерием фазового перехода только приближенно. Переходы второго рода связаны с потерей устойчивости по отношению к флуктуациям парных корреляций.
\end{abstract}

Ключевые слова: частичные распределения, диагонализация, устойчивость экстремума, фазовые переходы, критические точки.

\section{1. ВВЕДЕНИЕ}

Термодинамическая устойчивость системы и потеря этой устойчивости непосредственно связаны с возникновением различных фаз и переходами между ними. Проблемы существования различных фаз и фазовых переходов как следствия принципов классической статистической физики рассмотрены в ряде работ, довольно полный обзор которых приведен в монографии [1]. Наиболее прозрачная картина возникновения различных фаз и фазовых переходов дана, на наш взгляд, в книге [2]. Согласно этой работе статистическая сумма системы может быть представлена в виде контурного интеграла по активности, продолженной в комплексную плоскость. Вычисление этого интеграла проводится методом перевала. В случае существования нескольких точек перевала основной вклад вносит самый низкий перевал, а вклад других точек перевала экспоненциально мал и исчезает в термодинамическом пределе. Именно самая низкая точка перевала определяет термодинамические свойства

*Тюменский государственный университет, Тюмень, Россия.

E-mail: earin@utmn.ru 
стабильной фазы. Остальные точки перевала соответствуют метастабильным состояниям. Совпадение высот двух перевалов при изменении термодинамических параметров приводит к равновесию фаз и к фазовому переходу при дальнейшем изменении этих параметров. Однако метод перевала не получил дальнейшего развития по той причине, что, давая качественно правильную картину фазовых переходов, он мог служить основой конструктивного алгоритма вычисления термодинамических функций только в одном, давно и хорошо изученном случае - в случае вириального разложения для газа малой плотности.

В современной статистической физике широко используется другой метод - метод частичных функций распределения, который приводит, по существу, к идентичной картине равновесия фаз и фазовых переходов. Равновесные функции распределения (частичные плотности) определяются из условия минимума термодинамического потенциала как функционала от этих функций распределения. Если функционал имеет несколько экстремумов, то стабильное состояние определяется самым глубоким, глобальным, минимумом. Остальные минимумы вносят исчезающий в термодинамическом пределе вклад и описывают метастабильные состояния. Равновесие фаз отвечает одинаковой глубине соответствующих этим фазам минимумов, что совпадает с условием равенства химических потенциалов фаз. Дальнейшее изменение соотношения глубин минимумов соответствует фазовому переходу. Минимумы термодинамического потенциала в методе частичных функций распределения соответствуют точкам перевала в методе контурного интеграла.

И тот, и другой метод приводят к выводу, что термодинамические свойства системы определяются только стабильной фазой, а метастабильные состояния на равновесные свойства системы не влияют. Фазовый переход первого рода при таком подходе не связан с потерей термодинамической устойчивости стабильной фазы, ее свойства могут быть непрерывно продолжены в метастабильную область. Определение условия равновесия двух фаз возможно только при сравнении их термодинамических потенциалов - глубин двух минимумов или высот двух точек перевала. Приближенное определение параметров фазового равновесия по свойствам одной фазы - по одностороннему условию - возможно только в том случае, когда априори известно, что область метастабильных состояний этой фазы достаточно узка. Исчезновение точки перевала в методе контурного интеграла и минимума в методе частичных функций распределения соответствует спинодали, т.е. полной потере устойчивости, причем не стабильной, а метастабильной фазы. Идентичными являются описания критических точек в этих двух методах - критическая точка определяется слиянием двух точек перевала в одном методе и двух минимумов во втором.

Однако, несмотря на соответствие картин изменения фазовых состояний, математический аппарат этих методов отличается весьма существенно, в частности, метод частичных функций распределения дает описание структуры каждой фазы, которое принципиально не может быть получено в методе контурного интеграла. Это определяет более широкие возможности метода частичных функций распределения и объясняет причину его широкого распространения. В настоящей работе рассмотре- 
на связь условий устойчивости термодинамической системы со свойствами функций распределения (корреляционных функций), описывающих структуру этой системы. Мы используем обозначения работ [3], [4], продолжением которых является настоящая работа.

\section{2. КРИТЕРИЙ УСТОЙЧИВОСТИ}

Эффективным методом анализа совокупности частичных функций распределения (частичных плотностей) и корреляционных функций является метод производящего функционала. В настоящей работе используются свойства производящих функционалов, достаточно подробно описанные в работах [3], [4]. В работах [3] показано, что система уравнений для частичных плотностей эквивалентна вариационной задаче для термодинамического потенциала как функционала либо от всех плотностей, либо от корреляционных функций, и найдено явное выражение для этого функционала $A(R)$ через производящий функционал $R$ частичных плотностей. При этом $A(R)$ содержит вспомогательный функционал

$$
Q_{\text {in }}(\xi, \eta)=-D_{\rho}(\xi) D_{\rho}(\eta) A(R), \quad D_{\rho}(\xi)=\sum_{n} \int \prod_{n} \xi(n) \frac{\delta}{\delta \rho_{n}} d\{n\} .
$$

Индекс "in" указывает, что этот функционал относится к первоначальной формулировке теории.

Отсюда следует, что вторая вариация термодинамического потенциала в его первоначальной формулировке имеет вид

$$
\delta^{2} A=-\frac{1}{2} \delta R(\xi) \otimes Q_{\text {in }}(\xi, \eta) \otimes \delta R(\eta) .
$$

Рассматривать частичные плотности в качестве независимых переменных вариационной задачи неудобно, так как при этом необходимо учитывать нелинейные асимптотические условия ослабления корреляций, согласно которым частичная плотность группы частиц распадается на произведение частичных плотностей подгрупп частиц при удалении этих подгрупп друг от друга. Выбрав в качестве независимых переменных мультипликативные корреляции, обращающиеся в нуль при удалении на бесконечность хотя бы одной частицы из рассматриваемой группы, мы решим возникшую проблему. Выразим вариации плотностей через вариации корреляций:

$$
\begin{aligned}
\delta R(\xi) & =R(\xi \nu) \otimes R^{-1}(\nu \gamma) \otimes \delta R(\gamma)=R(\xi \nu) \otimes \delta L r(\nu)= \\
& =R(\xi \nu) \otimes \exp \left(\int \nu(q) d q\right) \delta \operatorname{Lm}(\nu)=R(\xi(\nu+1)) \otimes \delta L m(\nu),
\end{aligned}
$$

где $L r$ - производящий функционал функций $\ln \rho_{n} / \rho^{n}$ и $L m-$ производящий функционал функций $\ln \left(1+M_{n}\right), M_{n}$ - мультипликативные корреляции. Тогда вторая вариация термодинамического потенциала приобретает вид

$$
\begin{aligned}
& -\frac{1}{2} \delta \operatorname{Lm}(\nu) \otimes R((\nu+1) \xi) \otimes Q_{\text {in }}(\xi, \eta) \otimes R(\eta(\gamma+1)) \otimes \delta \operatorname{Lm}(\nu)= \\
& =\frac{1}{2} \delta \operatorname{Lm}(\nu) \otimes Q(\nu, \gamma) \otimes \delta \operatorname{Lm}(\gamma)
\end{aligned}
$$


где $Q$ - модифицированный производящий функционал прямых корреляций, содержащий разложение только по связанным диаграммам [4]. Функционалы $Q_{\text {in }}$ и $Q$ содержат обобщенные прямые корреляции и связаны с полными корреляциями (аддитивными или мультипликативными) обобщенным соотношением Орнштейна-Цернике: $Q_{\text {in }} \otimes P_{\text {in }}=Q \otimes P=I$. Явный вид функционалов $P_{\text {in }}$ и $P$ и вывод этого соотношения приведены в работах [3], [4]. При этом для удобства в определении функционала $Q$ изменен знак.

Очевидно, условие устойчивости фазы, определяемой локальным минимумом термодинамического потенциала $A$, сводится к требованию положительной определенности полученной квадратичной формы. Для анализа этого требования используем метод последовательной диагонализации производящего функционала прямых корреляционных функций [4].

Для выделения в произвольном производящем функционале членов определенного порядка введем наряду с функционалом $I(\xi \eta)=e^{(\xi \eta)}$, являющимся при свертке единичным оператором, ограничивающий функционал $I^{n}(\xi \eta)=\sum_{k=0}^{n}(\xi \eta)^{k} / k$ !, являющийся проекционным оператором, который выделяет члены порядка не выше $n$; положим $I_{m}^{n}=I^{n}-I^{m-1}$. Обозначения $B^{n}=B \otimes I^{n}, B_{m}^{n}=B \otimes I_{m}^{n}$ для соответствующих членов произвольного функционала $B$ очевидны.

Процедура последовательной диагонализации состоит в последовательном определении функционалов

$$
Q^{n}=Q^{n-1}+\left(I-Q^{n-1} \otimes P^{n}\right) \otimes \widetilde{Q}_{n}^{n} \otimes\left(P^{n} \otimes Q^{n-1}-I\right),
$$

где функционал $\widetilde{Q}_{n}^{n}$ определяется соотношениями [4]

$$
\widetilde{Q}_{n}^{n} \otimes \widetilde{P}_{n}^{n}=I_{n}^{n}, \quad \widetilde{P}_{n}^{n}=P^{n}-P^{n} \otimes Q^{n-1} \otimes P^{n} .
$$

Так как $P^{n}=P^{n-1}+P_{n}^{n}+P_{n-1}^{n}+P_{n}^{n-1}$, то очевидно, что функционалы $\widetilde{P}_{n}^{n}$ и $\widetilde{Q}_{n}^{n}$ содержат члены только порядка $n$ по каждой функции, от которой они зависят, функционал $Q^{n}$ содержит все члены функционала $Q$ порядка не больше $n$ и $Q^{n} \rightarrow Q$ при $n \rightarrow \infty$.

Рассмотрим квадратичную форму $n$-го порядка, определяющую условие устойчивости, с учетом последовательной диагонализации:

$$
\begin{aligned}
X^{n} \otimes Q^{n} \otimes X^{n}= & X^{n-1} \otimes Q^{n-1} \otimes X^{n-1}+\left(X^{n}-X^{n} \otimes Q^{n-1} \otimes P^{n}\right) \otimes \widetilde{Q}_{n}^{n} \otimes \\
& \otimes\left(X^{n}-P^{n} \otimes Q^{n-1} \otimes X^{n}\right)= \\
= & X^{n-1} \otimes Q^{n-1} \otimes X^{n-1}+X_{n}^{n} \otimes \widetilde{Q}_{n}^{n} \otimes X_{n}^{n},
\end{aligned}
$$

где $X_{n}^{n}=X^{n}-X^{n} \otimes Q^{n-1} \otimes P^{n}=X^{n}-X^{n-1} \otimes Q^{n-1} \otimes P^{n-1}=X^{n}-X^{n-1}$. Таким образом, условие $X^{n} \otimes Q^{n} \otimes X^{n}>0$ сводится к

$$
X^{n-1} \otimes Q^{n-1} \otimes X^{n-1}+X_{n}^{n} \otimes \widetilde{Q}_{n}^{n} \otimes X_{n}^{n}>0
$$

или, что эквивалентно, к условиям

$$
X^{n-1} \otimes Q^{n-1} \otimes X^{n-1}>0 ; \quad X_{n}^{n} \otimes \widetilde{Q}_{n}^{n} \otimes X_{n}^{n}>0 .
$$


Полагая $n=1,2, \ldots$, получим последовательность условий

$$
X_{n}^{n} \otimes \widetilde{Q}_{n}^{n} \otimes X_{n}^{n}>0, \quad n=1,2, \ldots,
$$

где произвольным функциям $X_{n}^{n} \neq 0$ можно придать смысл относительных флуктуаций: $X_{1}^{1}=\delta \rho / \rho ; X_{n}^{n}=\delta M_{n} /\left(1+M_{n}\right), n \geqslant 2$. При этом все свертки $X_{n}^{n} \otimes \widetilde{Q}_{n}^{n} \otimes X_{n}^{n}$ являются обычными скалярными произведениями, так как функционал $X_{n}^{n}$ содержит только одну функцию, а функционал $\widetilde{Q}_{n}^{n}$ - только одно ядро.

Если рассматривать ядро функционала $\widetilde{Q}_{n}^{n}$ как квадратную матрицу (дискретную или непрерывную - безразлично), то положительная определенность квадратичной формы $X_{n}^{n} \otimes \widetilde{Q}_{n}^{n} \otimes X_{n}^{n}$ означает, что все собственные значения этой матрицы положительны. В этом случае ядро функционала $\widetilde{P}_{n}^{n}$ является матрицей, обратной матрице $\widetilde{Q}_{n}^{n}$, и все ее собственные значения также положительны. Таким образом, мы приходим к альтернативной формулировке условия устойчивости

$$
X_{n}^{n} \otimes \widetilde{Q}_{n}^{n} \otimes \widetilde{P}_{n}^{n} \otimes \widetilde{Q}_{n}^{n} \otimes X_{n}^{n}=Y_{n}^{n} \otimes \widetilde{P}_{n}^{n} \otimes Y_{n}^{n}>0, \quad n=1,2, \ldots,
$$

где $Y_{n}^{n}=X_{n}^{n} \otimes \widetilde{Q}_{n}^{n}$. В представлении, в котором ядра функционалов $\widetilde{Q}_{n}^{n}$ и $\widetilde{P}_{n}^{n}$ являются диагональными матрицами, флуктуации $X_{n}^{n}$ и $Y_{n}^{n}$ совпадают с точностью до множителя (примером условий (13)-(15) следующего раздела может служить фурье-представление радиальных функций жидкости). В общем случае $X_{n}^{n}$ и $Y_{n}^{n}$ связаны линейным соотношением, вид которого определяется соотношением Орнштейна-Цернике между полными и прямыми корреляциями.

Известно [1], [5], что средние значения флуктуаций различных величин связаны с соответствующими восприимчивостями. Так, распределение флуктуаций макроскопических параметров является гауссовым, причем показатель экспоненты равен второй вариации термодинамического потенциала (с обратным знаком), содержащей сопряженные этим параметрам восприимчивости [6]. Изучение условий устойчивости по отношению к флуктуациям корреляций также подчеркивает эту связь.

\section{3. ПЕРВЫЙ ПОРЯДОК УСЛОВИЯ УСТОЙчИВОСТИ. ОДНОРОДНЫЕ СОСТОЯНИЯ}

Условия устойчивости первого порядка (по отношению к флуктуациям унарной плотности) налагают ограничения на свойства бинарной корреляции. В эти условия входит ядро функционала $\widetilde{Q}_{1}^{1}=Q_{1}^{1}=\rho_{1}(1) \delta(1,2)-\rho_{1}(1) \rho_{1}(2) C(1,2)$, где $C(1,2)-$ прямая корреляционная функция Орнштейна-Цернике [3], [4]. Ядро функционала $\widetilde{P}_{1}^{1}=P_{1}^{1}$ имеет вид $\delta(1,2) / \rho_{1}(1)+h_{2}(1,2)$, где $h_{2}(1,2)=M_{2}(1,2)$ - безразмерная аддитивная полная парная корреляционная функция, совпадающая с парной мультипликативной корреляционной функцией. Тогда условие устойчивости первого порядка принимает вид

$$
\int\left(X(1)\left(\rho_{1}(1) \delta(1,2)-\rho_{1}(1) \rho_{1}(2) C(1,2)\right) X(2)\right) d\{2\}>0,
$$


ТЕРМОДИНАМИЧЕСКАЯ УСТОЙЧИВОСТЬ, ОСОБЫЕ ТОЧКИ И ФАЗОВЫЕ ПЕРЕХОДЫ 517 а альтернативное условие - вид

$$
\int\left(Y(1)\left(\frac{\delta(1,2)}{\rho_{1}(1)}+h_{2}(1,2)\right) Y(2)\right) d\{2\}>0 .
$$

В случае пространственно однородной системы (жидкость или газ) $\rho_{1}=\rho=$ const. Преобразование Фурье в этом случае диагонализует ядра $Q_{1}^{1}, P_{1}^{1}$, и условие устойчивости приобретает вид

$$
\int\left(1-(2 \pi)^{3 / 2} \rho \widetilde{C}(k)\right)|\widetilde{X}(k)|^{2} d^{3} k>0,
$$

а альтернативное условие - вид

$$
\int\left(1+(2 \pi)^{3 / 2} \rho \tilde{h}(k)\right)|\tilde{Y}(k)|^{2} d^{3} k>0,
$$

откуда следует условие

$$
1-(2 \pi)^{3 / 2} \rho \widetilde{C}(k)=\left(1+(2 \pi)^{3 / 2} \rho \tilde{h}(k)\right)^{-1}>0
$$

(равенство двух выражений является соотношением Орнштейна-Цернике в фурьепредставлении). Граница устойчивости метастабильных однородных состояний, т.е. спинодаль, может определяться либо условием

$$
1-(2 \pi)^{3 / 2} \rho \widetilde{C}(k)=0
$$

либо условием

$$
1+(2 \pi)^{3 / 2} \rho \tilde{h}(k)=0 .
$$

При этом в окрестности границы, определяемой равенством (16), вторая вариация термодинамического потенциала мала, что может привести к малым изменениям унарной плотности. Из характерного вида прямой корреляционной функции [5], [7] следует, что равенство (16) первоначально будет достигнуто при $k=0$ (см. приложение), и это соответствует критической точке жидкость-пар, затем условие устойчивости (положительности вариации термодинамического потенциала) будет нарушаться при все более возрастающих значениях волнового числа $k$. Это можно интерпретировать как возникновение длинноволновой (макроскопической) неоднородности типа расслоения на две однородные фазы с малой разностью плотностей $\Delta \rho$, т.е. расслоения газа на жидкую и паровую фазы. При этом по мере удаления от критической точки, для которой $1-(2 \pi)^{3 / 2} \rho \widetilde{C}(0)=0$, возрастает разность $\Delta \rho$ плотностей фаз и расширяется область значений волновых чисел $k$, дающих вклад в неоднородность, т.е. межфазная переходная область по мере роста $\Delta \rho$ становится более резкой и узкой [8].

Для анализа условия (17) необходимо учесть, что $h(r) \approx-1$ при $r \leqslant r_{0}$, где $r_{0}-$ радиус “твердой сердцевины”, определяемый интенсивным отталкиванием атомов на малом расстоянии, а также что эта функция имеет сравнительно узкий и высокий положительный максимум при $r \approx r_{1}$, где $r_{1}$ соответствует минимуму энергии 
парного взаимодействия частиц. Кроме того,

$$
k \tilde{h}(k)=\left(\frac{2}{\pi}\right)^{1 / 2} \int_{0}^{\infty} h(r) \sin (k r) r d r
$$

откуда следует, что вклад максимума радиальной функции в функцию $k \tilde{h}(k)$ положителен при $k r_{1} \approx \pi / 2,5 \pi / 2, \ldots$ и отрицателен при $k r_{1} \approx 3 \pi / 2, \ldots$, что приводит к чередованию максимумов и минимумов этой функции, следовательно, условие (17) может быть достигнуто при $k r_{1} \approx 3 \pi / 2$.

Если $1+(2 \pi)^{3 / 2} \rho \tilde{h}(k)$ мало, то $1-(2 \pi)^{3 / 2} \rho \widetilde{C}(k)$ велико, и изменение термодинамического потенциала не может быть малым. Таким образом, условие (17) может быть достигнуто при значениях термодинамических параметров, при которых периодическая структура с периодом, приблизительно равным $r_{1}$, заведомо имеет существенно более низкий термодинамический потенциал, чем однородное состояние. Поэтому условие (17) можно в некотором приближении сопоставить условию кристаллизации только при довольно существенных оговорках.

Как уже отмечалось, равенства (16), (17) определяют границу устойчивости метастабильных состояний (кроме критической точки перехода жидкость-пар, которая согласно условию (16) лежит и на спинодали, и на бинодали). Любой "односторонний" (основанный на анализе свойств одной фазы без сравнения со свойствами других фаз) критерий фазового перехода не может быть точным, его можно использовать только как приближенный и только при наличии веских оснований полагать, что область метастабильных состояний достаточно узка. Равенство в условии (17) может быть достигнуто в результате понижения минимума фурье-образа парной корреляции, что означает повышение ее первого максимума и в координатном представлении, и в фурье-представлении. Поэтому, опираясь на условие (17), можно сделать вывод о том, что кристаллизация наступает при достаточно жесткой корреляции значений координат ближайших соседей. Конкретные значения высоты и ширины максимумов корреляционной функции и ее фурье-образа или глубины минимума ее фурье-образа в точке кристаллизации можно установить только при сравнении термодинамических функций жидкости и кристалла. При этом результаты, полученные для различных моделей или потенциалов, могут не совпадать.

\section{4. УСТОЙЧИВОСТЬ КРИСТАЛЛИЧЕСКОГО СОСТОЯНИЯ}

Для анализа устойчивости периодической кристаллической структуры примем модель сильно локализованных частиц. Это означает, что мы аппроксимируем унарную плотность выражением

$$
\rho_{1}(r)=(1-c) \sum_{l} \rho_{0}\left(r-r_{l}\right)
$$

где $r_{l}$ - радиус-вектор узла $l$ кристаллической решетки, $c$ - концентрация вакансий и $\rho_{0}-$ узкое нормированное распределение в окрестности узла. Для простоты 
рассмотрим структуру, содержащую одну частицу в элементарной ячейке. Обобщение на более сложные структуры в достаточной мере очевидно. Если в качестве единицы объема принять объем одной ячейки решетки, то средняя плотность равна вероятности $(1-c)$ заполнения ячейки. Бинарные корреляционные функции, и прямая, и полная, определяются их значениями в узлах решетки, которые можно интерпретировать также как средние значения по ячейкам. Инвариантность относительно сдвига на период решетки приводит к тому, что эти функции зависят от расстояния между узлами.

Флуктуации плотности при интегрировании усредняются по объему ячейки с весом $\rho_{0}$. В итоге условия устойчивости принимают вид

$$
\begin{aligned}
& \sum_{l_{1}, l_{2}} \overline{X\left(l_{1}\right) X\left(l_{2}\right)}\left(\delta\left(l_{1}, l_{2}\right)-(1-c) C\left(l_{1}-l_{2}\right)\right)= \\
& =(1-(1-c) C(0)) \sum_{l} \overline{\left(X_{l}-\bar{X}_{l}\right)^{2}}+ \\
& \quad+\sum_{l_{1}, l_{2}} \bar{X}\left(l_{1}\right) \bar{X}\left(l_{2}\right)\left(\delta\left(l_{1}, l_{2}\right)-(1-c) C\left(l_{1}-l_{2}\right)\right)>0 \\
& \sum_{l_{1}, l_{2}} \overline{Y\left(l_{1}\right) Y\left(l_{2}\right)}\left(\delta\left(l_{1}, l_{2}\right)+(1-c) h\left(l_{1}-l_{2}\right)\right)= \\
& =(1+(1-c) h(0)) \sum_{l} \overline{\left(Y_{l}-\bar{Y}_{l}\right)^{2}}+ \\
& \quad+\sum_{l_{1}, l_{2}} \bar{Y}\left(l_{1}\right) \bar{Y}\left(l_{2}\right)\left(\delta\left(l_{1}, l_{2}\right)+(1-c) h\left(l_{1}-l_{2}\right)\right)>0 .
\end{aligned}
$$

При этом соотношение Орнштейна-Цернике для функций $C(l)$ и $h(l)$ принимает вид

$$
h(l)-C(l)-(1-c) \sum_{l^{\prime}} h\left(l^{\prime}\right) C\left(l-l^{\prime}\right)=0 .
$$

Все соотношения на решетке можно диагонализовать преобразованием Фурье

$$
\widetilde{F}(k)=\sum_{l} F(l) e^{i k r_{l}}, \quad F(l)=\frac{1}{B} \int_{B} \widetilde{F}(k) e^{-i k r_{l}} d^{3} k,
$$

где $B=(2 \pi)^{3}$ - объем ячейки обратной решетки или зоны Бриллюэна. В результате такого преобразования получим

$$
(1+(1-c) \tilde{h}(k))(1-(1-c) \widetilde{C}(k))=1,
$$

и условие устойчивости принимает вид

$$
\int_{B}(1-(1-c) \widetilde{C}(k))|\phi(k)|^{2} d^{3} k>0
$$

где $\phi(k)=\sum_{l} X(l) e^{i k r_{l}}$. При выполнении этого условия

$$
\int_{B}(1-(1-c) \widetilde{C}(k)) d^{3} k=B(1-(1-c) C(0))>0,
$$


следовательно, множитель при первой сумме и весь первый член в правой части равенства в условии (20) положительны.

Если дополнительно учесть, что $h(0)=-1$ (в силу непроницаемости частиц), то $\tilde{h}(k)=-1+\tilde{h}_{1}(k)$, где функция $\tilde{h}_{1}(k)$ определяется рядом Фурье без постоянного члена. Таким образом, условие устойчивости можно представить в виде

$$
1-(1-c) \widetilde{C}(k)=\left(c+(1-c) \tilde{h}_{1}(k)\right)^{-1}>0 .
$$

Отсюда следует, что $c /(1-c)>\left|\tilde{h}_{1}(k)\right|$. Сопоставим это соотношение с интегралом сжимаемости $\left(\rho k_{\mathrm{B}} T \chi\right)$, который для нашей модели имеет вид

$$
1+\int h(r) \rho_{1}(r) d^{3} r=1+(1-c) \sum_{l} h(l)=\frac{1}{B} \int_{B}\left(c+(1-c) \tilde{h}_{1}(k)\right) d^{3} k .
$$

Очевидно, что условие устойчивости кристалла совпадает с условием положительности его изотермической сжимаемости $\chi$. Отсюда следует, в частности, что бездефектный кристалл не может иметь конечную сжимаемость, а условие $\chi \rightarrow 0$ нарушения устойчивости для плотноупакованной структуры недостижимо при ограниченном давлении (не изменяющем электронную структуру атомов и характер их связи).

Существует мнение, что потеря устойчивости кристалла определяется увеличением концентрации вакансий при уменьшении плотности. Однако получить такое заключение на основании неравенства (26) не представляется возможным. Более естественной является модель плавления кристалла в результате локального перехода плотноупакованной структуры в более рыхлую при повышении температуры [9].

\section{5. УСЛОВИЕ УСТОЙЧИВОСТИ ВТОРОГО ПОРЯДКА}

Условия устойчивости второго порядка (устойчивости по отношению к флуктуациям парной корреляции) налагают ограничения на свойства корреляционных функций до четвертого порядка. Рассмотрим эти условия, полагая, что условие устойчивости первого порядка выполнено.

Явное выражение для функционала $P$ было получено в работе [3]:

$$
P(\xi, \eta)=R^{-1}(\xi \eta)-1+P_{1}(\xi, \eta)
$$

где

$$
P_{1}(\xi, \eta)=R^{-1}(\xi \eta(1+\gamma)) \bigotimes_{\gamma}\left(e^{G(\xi+\eta+\gamma)-G(\xi+\gamma)-G(\eta+\gamma)+G(\gamma)}-1\right) .
$$

С точностью до второго порядка по функциям $\xi$ и $\eta$ имеем

$$
\begin{aligned}
\left(P_{1}\right)^{2}=\int & h_{2}(1,2) \xi(1) \eta(2) d(1,2)+\frac{1}{2} \int h_{3}(1,2,3) \xi(1) \eta(2)(\xi(3)+\eta(3)) d(1,2,3)+ \\
& +\int h_{3}(1,2,3) \xi(1) \eta(2) \frac{\xi(3) \eta(3)}{\rho_{1}(3)} d(1,2,3)+
\end{aligned}
$$




$$
+\frac{1}{4} \int\left(h_{4}(1,2,3,4)+2 h_{2}(1,2) h_{2}(3,4)\right) \xi(1) \eta(2) \xi(3) \eta(4) d(1,2,3,4) .
$$

Отсюда получим выражение для функционала $\widetilde{P}_{2}^{2}(\xi, \eta)$ :

$$
\begin{aligned}
\widetilde{P}_{2}^{2}(\xi, \eta)=\frac{1}{2} & \int h_{3}(1,3,5) Q_{1}^{1}(5,6) h_{3}(2,4,6) \xi(1) \xi(2) \eta(3) \eta(4) d\{6\}+ \\
& +\frac{1}{2} \int \frac{\xi(1) \xi(2) \eta(1) \eta(2)}{\rho_{2}(1,2)} d\{2\}+\frac{1}{2} \int h_{3}(1,2,3) \xi(1) \eta(2) \frac{\xi(3) \eta(3)}{\rho_{1}(3)} d\{3\}+ \\
& +\frac{1}{4} \int\left(h_{4}(1,2,3,4)+2 h_{2}(1,2) h_{2}(3,4)\right) \xi(1) \eta(2) \xi(3) \eta(4) d\{4\}
\end{aligned}
$$

Рассмотрим условие устойчивости в форме $Y_{2}^{2} \otimes \widetilde{P}_{2}^{2} \otimes Y_{2}^{2}>0$. Очевидно, неравенство $\int(Y(1,2))^{2} / \rho_{2}(1,2) d\{2\}>0$ выполняется при $\rho_{2}>0$. Этот член является второй вариацией двухчастичной энтропии $\int \rho_{2} \ln \rho_{2} d\{2\}$, выпуклой вниз при всех неотрицательных значениях $\rho_{2}$. При этом нулевые или очень малые значения бинарной плотности соответствуют области твердой сердцевины, корреляционная функция в этой области не флуктуирует, поэтому рассматриваемый член остается всегда конечным.

Выражение $\int h_{3}(1,3,4) Y(3,4) d(3,4)=\widetilde{X}(1)$ при произвольной $Y(3,4)$ можно рассматривать как произвольную функцию, откуда следует, что

$$
\int \tilde{X}(1) Q_{1}^{1}(1,2) \widetilde{X}(2) d(1,2)>0
$$

в силу условия устойчивости первого порядка. Таким образом, остается условие

$$
\begin{gathered}
\frac{1}{4} \int\left(h_{4}(1,2,3,4)+2 h_{2}(1,2) h_{2}(3,4)\right) Y(1,3) Y(2,4) d\{4\}+ \\
+\int h_{3}(1,2,3) \frac{Y(1,2) Y(1,3)}{\rho_{1}(1)} d\{3\}>0 .
\end{gathered}
$$

Сопоставим полученное неравенство и условия на теплоемкость [3]. Для случая системы с бинарным взаимодействием теплоемкость задается соотношением

$$
\begin{aligned}
2 k T^{2}\left(C_{V}-C_{V 0}\right)= & \int \rho_{2}(1,2) \phi^{2}(1,2) d\{2\}+\int \rho_{3}(1,2,3) \phi(1,2) \phi(1,3) d\{3\}+ \\
& +\int\left(\rho_{4}(1,2,3,4)-\rho_{2}(1,2) \rho_{2}(3,4)\right) \phi(1,2) \phi(3,4) d\{4\} .
\end{aligned}
$$

При этом

$$
\begin{aligned}
& \frac{\rho_{4}(1,2,3,4)-\rho_{2}(1,2) \rho_{2}(3,4)}{\prod_{i} \rho_{1}(i)}=h_{4}(1,2,3,4)+ \\
& +h_{2}(1,3) h_{2}(2,4)+h_{2}(2,3) h_{2}(1,4)+\sum h_{3}(i, j, k) .
\end{aligned}
$$


Таким образом, условие устойчивости второго порядка фактически совпадает с условием положительности и конечности теплоемкости. Нарушение этого условия и расходимость выражения для теплоемкости соответствуют тому, что одно или несколько собственных значений непрерывной матрицы $\widetilde{Q}_{2}^{2}$ обращаются в нуль. Это происходит либо в точке фазового перехода второго рода, либо в лямбда-точке, где теплоемкость обращается в бесконечность.

Исследование устойчивости по отношению к флуктуациям корреляций более высоких порядков может быть проведено аналогично, но оказывается более громоздким, а результаты такого исследования - не столь наглядными.

\section{ПРИЛОЖЕНИЕ}

Граница устойчивости жидкости определяется аналитическими свойствами фурье-образов радиальной (полной) $g(r)$ и прямой $C(r)$ корреляционных функций, связанных соотношением Орнштейна-Цернике. В фурье-представлении это соотношение имеет вид

$$
\left(1-(2 \pi)^{3 / 2} \rho \widetilde{C}(k)\right)\left(1+(2 \pi)^{3 / 2} \rho \tilde{h}(k)\right)=1 .
$$

Для анализа свойств корреляционных функций удобно воспользоваться разложением функций $g(r)$ и $C(r)$ по функциям $\psi_{t}(r)=e^{-t r} /(t r)$, осуществляющим представление группы движения трехмерного евклидова пространства [10]. Система этих функций является полной в пространстве радиальных функций, любая такая функция и ее фурье-образ представимы в виде

$$
f(r)=\int_{0}^{\infty} \bar{f}(t) \psi_{t}(r) d t, \quad \tilde{f}(k)=\int_{0}^{\infty} \frac{\bar{f}(t) d t}{t\left(t^{2}+k^{2}\right)}
$$

соответственно. Другими словами, $\bar{f}(t)$ является спектральной плотностью полюсов фурье-образа рассматриваемой функции. В теории жидкости полную радиальную функцию $g(r)$ иногда представляют не в виде интеграла, а в виде $\sum_{n} a_{n} \psi\left(t_{n} r\right)$, причем значения $t_{n}$ попарно сопряжены. Очевидно, что такая замена интеграла суммой не влияет на окончательные выводы.

Функция $r g(r)=\int_{0}^{\infty} \bar{g}(t) \frac{e^{-t r}}{t} d t$ конечна при любых $r$, в том числе при $r=0$, следовательно, интеграл $\int_{0}^{\infty} \bar{g}(t) / t d t$ сходится как на верхнем, так и на нижнем пределах. Отсюда функция $\tilde{g}(k)=\int_{0}^{\infty} \frac{\bar{g}(t) d t}{t\left(t^{2}+k^{2}\right)}$ конечна при любом конечном значении $k$. Поэтому функция $g(k)$ может обращаться в бесконечность только при $k=0$, при этом выполняется условие (16), определяющее критическую точку и спинодаль системы жидкость-пар. Характер возникающей особенности подробно рассмотрен в работе [11]. Кроме того, на примере квазикристаллической модели можно убедиться, что при некоторых конечных значениях $k$ возможно (реально недостижимое) формальное выполнение условия (17). Других нулей и особенностей, определяемых условиями (16), (17), фурье-образы радиальных функций жидкости не имеют. 
ТЕРМОДИНАМИЧЕСКАЯ УСТОЙЧИВОСТЬ, ОСОБЫЕ ТОЧКИ И ФАЗОВЫЕ ПЕРЕХОДЫ 523

\section{Список литературы}

[1] К. Хуанг, Статистическая механика, Мир, М., 1966.

[2] Б. Т. Гейликман, Статистическая теория фазовых превращений, ГИТТЛ, М., 1954.

[3] Э. А. Аринштейн, ТМФ, 124:1 (2000), 136-147; 143:1 (2005), 150-160.

[4] Э. А. Аринштейн, Р. М. Ганопольский, ТМФ, 131:2 (2002), 278-287.

[5] Р. Балеску, Равновесная и неравновесная статистическая механика, т. 1, Мир, М., 1977.

[6] Л. Д. Ландау, Е. М. Лифшиц, Теоретическая физика Т. V. Статистическая физика, Наука, М., 1976.

[7] К. Крокстон, Физика жидкого состояния. Статистическое введение, Мир, М., 1978.

[8] Э. А. Аринштейн, ТМФ, 148:2 (2006), 323-336.

[9] Э. А. Аринштейн, ТМФ, 151:1 (2007), 155-171.

[10] Н.Я. Виленкин, Специальные функиии и теория представлений групп, Наука, М., 1965.

[11] Э.А.Аринштейн, ТМФ, 138:1 (2004), 127-138.

Поступила в редакцию 19.04.2007, после доработки 9.06.2007 